\title{
Intuitionistic Fuzzy Simplex Method
}

\author{
R.Parvathi \\ Department of Mathematics, Vellalar Collegefor Women, \\ Erode, Tamilnadu, India
}

\author{
C.Malathi \\ Department of Mathematics, Gobi Arts and Science \\ College, Gobi, Tamilnadu, India
}

\begin{abstract}
This paper deals with Intuitionistic Fuzzy Linear Programming Problems(IFLPPs) using Symmetric Trapezoidal Intuitionistic Fuzzy Numbers(STIFNs) and the arithmetic operations defined on them. A special ranking function is presented in project environment to rank STIFN. An attempt has been made to solve the given IFLPP without converting them to crisp linear programming problems by using intuitionistic fuzzy simplex method and the algorithm is effective and reasonable as is evident from the results of a numerical example.
\end{abstract}

\section{General Terms}

Decision Making, Intuitionistic Fuzzy Optimization.

\section{Keywords}

Intuitionistic Fuzzy Set, Intuitionistic Fuzzy Linear Programming Problems, Intuitionistic Fuzzy Simplex Method, Symmetric Trapezoidal Instuitionistic Fuzzy Numbers, Ranking Function.

\section{INTRODUCTION}

In today's dynamic and unpredictable business environment, companies face the tremendous challenge of expanding markets and rising customer expectations. This compels them to lower total costs in the entire supply chain, reduce inventories, expand product choice, improve quality and efficient coordinate globe demand, supply and production.

In real life, due to the inevitable measurement

inaccuracy, the exact values of the measured quantities are not known and so the parameters of the problem are usually defined by the decision maker in an uncertain way. Therefore, it is desirable to consider the knowledge of experts about the parameters as fuzzy data.

Bellman and Zadeh(1970) [1] proposed the concept of decision making in fuzzy environments. By adopting this concept to a mathematical programming problem, Tanaka and Asai [2] proposed a formuation of fuzzy linear programming with fuzzy constraints and gave a method for its solution which bases on inequality relations between fuzzy numbers. Zimmermann [3] proposed the first formulation of Fuzzy Linear Programming Problem(FLPP).

The most common concept used in almost all these studies is ranking of fuzzy numbers. In order to rank fuzzy numbers, one fuzzy number needs to be compared with the others, but it is difficult to determine clearly which of them is larger or smaller. Numerous methods have been proposed in literature to rank fuzzy numbers $[4,5,6]$.

Though fuzziness in decision making problems has been studied by various researchers who viewed the goals as fuzzy sets, they are very limited in scope and in many cases, do not represent the real problem very well. In practice, due to insufficiency of the information available, it is not easy to describe the fuzzy constraint conditions by ordinary fuzzy sets and the evaluation of membership and non-membership values upto decision maker's satisfaction is not always possible.

Consequently, there remains an indeterministic part of which hesitation survives. In such situations, the Intuitionistic Fuzzy Set(IFS) theory introduced by Atanassov [7] seems to be applicable to address this issue of uncertainty. Burillo et al [8] proposed the definition of intuitionistic fuzzy number and studied perturbations of intuitionistic fuzzy number and the properties of the correlation between these numbers. On the front of ranking intuitionistic fuzzy numbers, some work has been reported in the literature. Mitchell [9] considered the problem of ranking a set of intuitionistic fuzzy numbers to define a fuzzy rank and a characteristic vagueness factor for each intuitionistic fuzzy number.

Kumar et al [10] introduced a new method for solving fully fuzzy linear programming problems with equality constraints by using ranking function with triangular fuzzy numbers. Maleki et al $[5,11]$ also employed the method of ranking functions for solving fuzzy linear programming problems.

K.Ganesan and P.Veeramani [12] introduced a new type of fuzzy arithmetic for symmetric trapezoidal fuzzy numbers and proposed a method for solving fuzzy linear programming problems. B.S.Mahapatra and G.S.Mahapatra [13] have presented intuitionistic fuzzy fault tree analysis using trapezoidal intuitionistic fuzzy number.

This paper introduces a new concept to the optimization problem in an IF environment and a special ranking function to rank STIFN and explains a solution procedure for IFLPP in which the right hand side constants are STIFN by using Intuitionistic Fuzzy Simplex Method.

This paper is organized as follows:

Section 2 briefly describes the basic definitions and notations of IFS, IFN, TIFN, FLPP and STIFN. A special ranking function is introduced in Section 3. Section 4 deals with a general Method of IFLPP. An IF Simplex Method has been introduced to solve the problem without converting it to its crisp equivalent and the application of this method is illustrated in Section 5. Finally, the paper is concluded in Section 6. 


\section{PRELIMINARIES}

In this section, some basic concepts and preliminary work required for the current work are dicussed.

Definition 2.1

An Intuitionistic Fuzzy Set (IFS) [7] $A$ in $X$ is defined as an object of the form $\mathrm{A}=$ $\left\{<x, \mu_{A}(x), v_{A}(x)>: x \in X\right\}$ where the functions $\mu_{A}: X \rightarrow[0,1]$ and $v_{A}: X \rightarrow[0,1]$ define the degree of membership and the degree of non-membership of the element $x \in X$ respectively, and for every $\mathrm{x} \in \mathrm{X}$ in $\mathrm{A}$, $0 \leq \mu_{A}(x)+v_{A}(x) \leq 1$ holds.

Definition 2.2

For every common fuzzy subset A on $\mathrm{X}$, Intuitionistic Fuzzy Index of $x$ in $A$ [7] is defined as $\pi_{A}(x)=1-\mu_{A}(x)-v_{A}(x)$. It is also known as degree of hesitancy or degree of uncertainty of the element $x$ in A.Obviously, for every $x \in X, 0 \leq \pi_{A}(x) \leq 1$.

Definition 2.3

An Intuitionistic Fuzzy Number (IFN)

[13] $\tilde{A}^{I}$ is

i) an intuitionistic fuzzy subset of the real line,

ii) normal, that is, there is some $x_{0} \in R$ such that $\mu_{\tilde{A}^{I}}\left(x_{0}\right)=1, v_{\tilde{A}^{I}}\left(x_{0}\right)=0$,

iii) convex for the membership function $\mu_{\tilde{A}^{I}}(x)$, that is,

$\mu_{\tilde{A}^{I}}\left(\lambda x_{1}+(1-\lambda) x_{2}\right)$

$\geq \min \left(\mu_{\tilde{A}^{I}}\left(x_{1}\right), \mu_{\tilde{A}^{I}}\left(x_{2}\right)\right)$,

for every $x_{1}, x_{2} \in R, \quad \lambda \in[0,1]$,

iv) concave for the non-membership function $\tilde{x}^{I}$ that is,

$v_{\tilde{A}^{I}}\left(\lambda x_{1}+(1-\lambda) x_{2}\right) \leq$

$\max \left(v_{\tilde{A}^{I}}\left(x_{1}\right), v_{\tilde{A}^{I}}\left(x_{2}\right)\right)$,

for every $x_{1}, x_{2} \in R, \quad \lambda \in[0,1]$.

Definition 2.4

A Trapezoidal Intuitionistic Fuzzy Number(TIFN) [13] $\tilde{A}^{I}$ is an IFS in $\mathrm{R}$ with membership function and nonmembership function as follows:

$\mu_{A} I(x)=\left\{\begin{array}{cc}\frac{x-\left(a_{1}-\alpha\right)}{\alpha} & \text { for } x \in\left[a_{1}-\alpha, a_{1}\right] \\ 1 & \text { for } x \in\left[a_{1}, a_{2}\right] \\ \frac{a_{2}+\beta-x}{\beta} & \text { for } x \in\left[a_{2}, a_{2}+\beta\right] \\ 0 & \text { otherwise }\end{array}\right.$

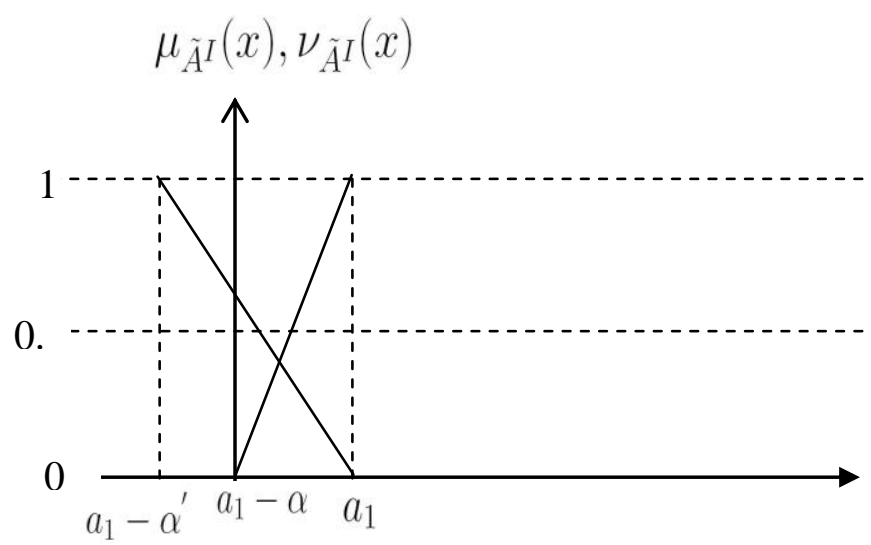

Fig 1: Diagrammatic representation of a TIFN

\section{Definition 2.5}

The support of an IFS $\tilde{A}^{I}$ on $\mathrm{R}$ is the crisp set of all $x \in R$ such that $\mu_{A^{A} I}(x)>0, \quad v_{A}(x)>0$ and

$v_{A^{I}}(x)=\left\{\begin{array}{cc}\frac{a_{1}-x}{\alpha^{\prime}} & \text { for } x \in\left[a_{1}-\alpha^{\prime}, a_{1}\right] \\ 0 & \text { for } x \in\left[a_{1}, a_{2}\right] \\ \frac{x-a_{2}}{\beta^{\prime}} & \text { for } x \in\left[a_{2}, a_{2}+\beta^{\prime}\right] \\ 1 & \text { otherwise }\end{array}\right.$

where $a_{1} \leq a_{2}, \quad \alpha, \beta \geq 0$ such that $\alpha \leq \alpha$ and $\beta \leq \beta$.

A TIFN is denoted by $\tilde{A}_{\text {TIFN }}^{I}=\left[a_{1}, a_{2}, \alpha, \beta ; a_{1}, a_{2}, \alpha^{\prime}, \beta^{\prime}\right]$

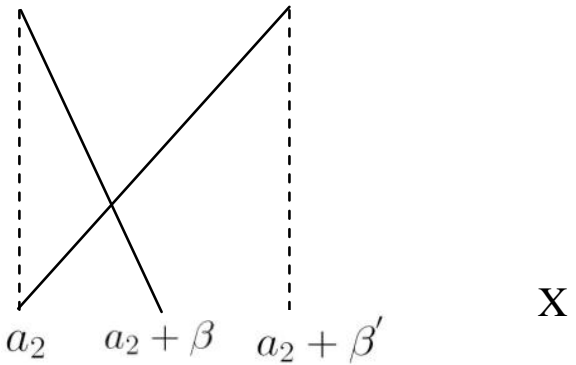

$$
\mu_{\nexists} I(x)+v_{\square_{A} I}(x) \leq 1 .
$$

Definition 2.6

An IFS $\mathrm{A}=\left\{\left\langle x, \mu_{A}(x), v_{A}(x)\right\rangle: x \in X\right\}$ is called IF-normal [14] if there exist atleast two points $x_{0}, x_{1} \in X$ such that $\mu_{A}\left(x_{0}\right)=1, v_{A}\left(x_{1}\right)=1$.

Therefore, a given intuitionistic fuzzy set A is IF-normal if there is atleast one point that surely belongs to $\mathrm{A}$ and atleast one point which does not belong to $\mathrm{A}$. 
Definition 2.7

A linear programming problem with fuzzy right hand side constants (FLPP) is defined as

Maximize $z=\sum_{j=1}^{n} c_{j} x_{j}$

subject to $\sum_{j=1}^{n} a_{i j} x_{j} \leq \tilde{b}_{i}, 1 \leq i \leq m$

$$
x_{j} \geq 0,1 \leq j \leq n
$$

where atleast one $x_{j}>0$ and $\tilde{b}$ is a fuzzy number and $x \in R$.

\section{Definition 2.8}

An IFS $\tilde{A}^{I}$ in $\mathrm{R}$ is said to be a Symmetric Trapezoidal Intuitionistic Fuzzy Number (STIFN) [15] if there exist real numbers $a_{1}, a_{2}, h, h^{\prime}$ where $a_{1} \leq a_{2}, h \leq h^{\prime}$ and $h, h^{\prime}>0$ such that the membership and non-membership functions are as follows:

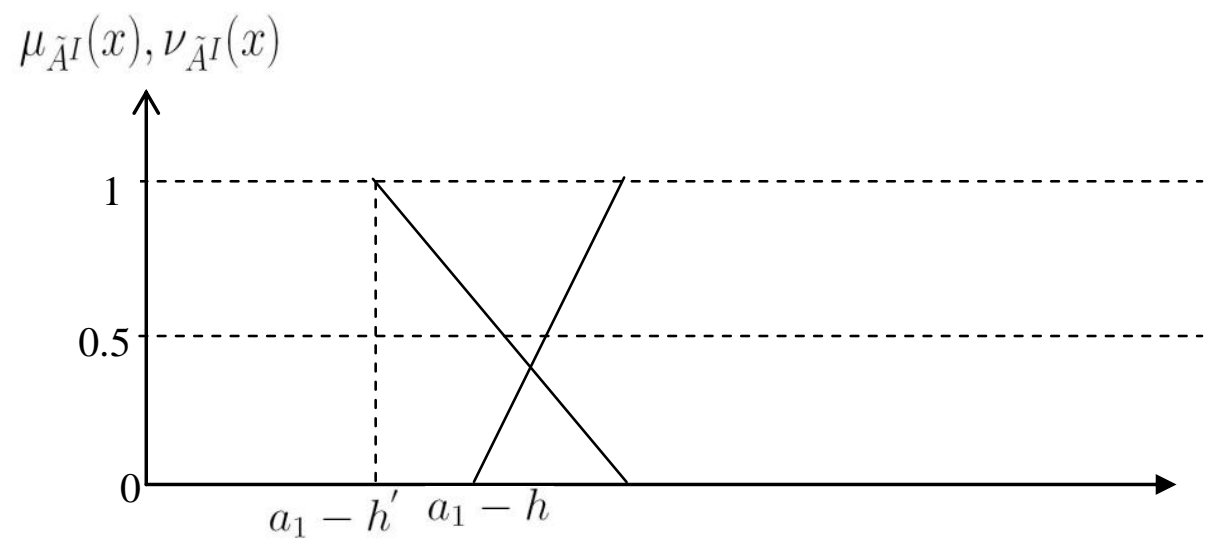

Fig 2: Diagrammatic representation of a STIFN

$\mu_{\nexists I}(x)=\left\{\begin{array}{cc}\frac{x-\left(a_{1}-h\right)}{h} & \text { for } x \in\left[a_{1}-h, a_{1}\right] \\ 1 & \text { for } x \in\left[a_{1}, a_{2}\right] \\ \frac{a_{2}+h-x}{h} & \text { for } x \in\left[a_{2}, a_{2}+h\right] \\ 0 & \text { otherwise }\end{array}\right.$

and

$v_{A^{\prime} I}(x)=\left\{\begin{array}{cc}\frac{a_{1}-x}{h^{\prime}} & \text { for } x \in\left[a_{1}-h^{\prime}, a_{1}\right] \\ 0 & \text { for } x \in\left[a_{1}, a_{2}\right] \\ \frac{x-a_{2}}{h^{\prime}} & \text { for } x \in\left[a_{2}, a_{2}+h^{\prime}\right] \\ 1 & \text { otherwise }\end{array}\right.$

where $\tilde{A}^{I}=\left[a_{1}, a_{2}, h, h ; a_{1}, a_{2}, h^{\prime}, h^{\prime}\right]$. 


\section{RANKING FUNCTION}

A convenient approach for solving IFLPP is based on the concept of comparision of IF numbers by use of ranking functions. A ranking function is used to order IFN and it is a mapping from an IFS to the set of real numbers R.

In fact, an efficient approach for ordering the elements of $\mathrm{F}(\mathrm{R})$, the set of STIFNs on $\mathrm{R}$, is to define a ranking function $\mathfrak{R}: F(R) \rightarrow R$ which maps each STIFN into the real line. Orders on $\mathrm{F}(\mathrm{R})$ are defined as follows:

$\tilde{A}^{I} \succeq \tilde{B}^{I}$ if and only if $\mathfrak{R}\left(\tilde{A}^{I}\right) \geq \mathfrak{R}\left(\tilde{B}^{I}\right)$

$\tilde{A}^{I} \succ \tilde{B}^{I}$ if and only if $\mathfrak{R}\left(\tilde{A}^{I}\right)>\mathfrak{R}\left(\tilde{B}^{I}\right)$

$\tilde{A}^{I} \approx \tilde{B}^{I}$ if and only if $\mathfrak{R}\left(\tilde{A}^{I}\right)=\mathfrak{R}\left(\tilde{B}^{I}\right)$ where

$\tilde{A}^{I}, \tilde{B}^{I}$ are in $\mathrm{F}(\mathrm{R})$.

Also, $\tilde{A}^{I} \preceq \tilde{B}^{I}$ if and only if $\mathfrak{R}\left(\tilde{B}^{I}\right) \geq \mathfrak{R}\left(\tilde{A}^{I}\right)$.

For any two STIFNs

$\tilde{A}^{I}=\left[a_{1}, a_{2}, h, h ; a_{1}, a_{2}, h^{\prime}, h^{\prime}\right] \quad$ and

$\tilde{B}^{I}=\left[b_{1}, b_{2}, k, k ; b_{1}, b_{2}, k^{\prime}, k^{\prime}\right]$, the relation $\preceq$ is a

partial order relation and is defined as $\tilde{A}^{I} \preceq \tilde{B}^{I}$ if and only if either $a_{1}+a_{2}+\frac{1}{2}\left(h^{\prime}-h\right)<b_{1}+b_{2}+\frac{1}{2}\left(k^{\prime}-k\right)$ (In this case, it can be written as $\left.\tilde{A}^{I} \prec \tilde{B}^{I}\right) \quad$ or $a_{1}+a_{2}+\frac{1}{2}\left(h^{\prime}-h\right)=b_{1}+b_{2}+\frac{1}{2}\left(k^{\prime}-k\right)$ (In this case, it can be written as $\tilde{A}^{I} \approx \tilde{B}^{I}$ and $\tilde{A}^{I}$ and $\tilde{B}^{I}$ are said to be equivalent.)

The following lemma is now immediate:

Lemma 3.1

Let $\mathfrak{R}$ be any linear ranking function. Then

(i) $\tilde{A}^{I} \succeq \tilde{B}^{I}$ if and only if $\tilde{A}^{I}-\tilde{B}^{I} \succeq 0$ iff $-\tilde{B}^{I} \succeq-\tilde{A}^{I}$

(ii) If $\tilde{A}^{I} \succeq \tilde{B}^{I}$ and $\tilde{C}^{I} \succeq \tilde{D}^{I}$, then $\tilde{A}^{I}+\tilde{C}^{I} \succeq \tilde{B}^{I}+\tilde{D}^{I}$.

Since there are many ranking functions for comparing IF numbers, a linear ranking function is introduced in this paper which is actually a ranking function $\mathfrak{R}$ such that

$$
\mathfrak{R}\left(K \tilde{A}^{I}+\tilde{B}^{I}\right)=K \mathfrak{R}\left(\tilde{A}^{I}\right)+\mathfrak{R}\left(\tilde{B}^{I}\right)
$$

Below a method of ranking STIFN is suggested which is actually a direct method for ranking classical intuitionistic fuzzy numbers.

$$
\mathfrak{R}\left(\tilde{A}^{I}\right)=a_{1}+a_{2}+\frac{1}{2}\left(h^{\prime}-h\right)
$$

where $\tilde{A}^{I}=\left[a_{1}, a_{2}, h, h ; a_{1}, a_{2}, h^{\prime}, h^{\prime}\right] \in F(R)$.

\section{INTUITIONISTIC FUZZY LINEAR PROGRAMMING PROBLEMS}

In the framework of IFLPP, there are no additional assumptions about the nature of objective functions and constraints. According to different hypothesis being considered, distinct IFLP problems are obtained.

If the decision maker does not know exactly the values of the decision making variables and the right hand side constants taking part in the problem (in this paper $x_{j}$ 's and $b_{j}$ 's because $a_{i j}$ 's and $c_{j}$ 's are supposed to be fixed throughout) and moreover, the vagueness is not of probabilistic kind, the inexact values can be determined by means of models using IF numbers. Here, the general form of such an IFLPP is considered and the solution procedure is illustrated.

Definition 4.1

An Intuitionistic Fuzzy Linear Programming Problem (IFLPP) is defined as

$$
\begin{gathered}
\operatorname{Max} \tilde{z}^{I} \approx c \tilde{x}^{I} \\
\text { subject to } A \tilde{x}^{I} \preceq \tilde{b}^{I} \\
\tilde{x}^{I} \succeq 0
\end{gathered}
$$

where $\tilde{b}^{I} \in(F(R))^{m}, \tilde{x}^{I} \in(F(R))^{n}$,

$A \in R^{m \times n}, c^{T} \in R^{n}$ and $\mathfrak{R}$ is a linear ranking function and $\mathrm{F}(\mathrm{R})$ is the set of STIFNs.

Definition 4.2

Any vector $\tilde{x}^{I} \in(F(R))^{n}$ which satisfies the constraints and non negative restrictions of (1) is said to be an 'intuitionistic fuzzy feasible solution'.

Definition 4.3

Let $\mathrm{S}$ be the set of all intuitionistic fuzzy feasible solutions of (1). Any vector $x_{0} \in S$ is said to be an 'intuitionistic fuzy optimum solution' to (1) if $c x_{0} \succeq c x$ for all $x \in S$ where

$$
c=\left(c_{1}, c_{2}, \ldots, c_{n}\right)
$$

and

$c x=c_{1} x_{1}+c_{2} x_{2}+\ldots+c_{n} x_{n}$.

Definition 4.4

For the IFLPP which is defined in (1), consider the system $A \tilde{x}^{I} \preceq \tilde{b}^{I}$ and $\tilde{x} \succeq 0$. Let $A=\left[a_{i j}\right]_{m \times n}$. Assume $\operatorname{rank}(\mathrm{A})=\mathrm{m}$. Partition $\mathrm{A}$ as $[\mathrm{B} \mathrm{N}]$ where $\mathrm{B}$ is non singular matrix of order $m \times m$. Therefore, $\operatorname{rank}(\mathrm{B})=\mathrm{m}$. Let $y_{j}$ be the solution to $B_{y}=a_{j}$.

It is apparent that the basic solution $\tilde{x}_{B}^{I}=\left(\tilde{x}_{B_{1}}^{I}, \ldots, \tilde{x}_{B_{m}}^{I}\right) \approx B^{-1} \tilde{b}^{I}, \tilde{x}_{N}^{I} \approx 0$ is a solution of $A \tilde{x}^{I} \approx \tilde{b}^{I}$. Here $\tilde{x}^{I}$ which is partitioned as $\left(\tilde{x}_{B}^{I}, \tilde{x}_{N}^{I}\right)^{T}$ is called an intuitionistic fuzzy basic solution corresponding to the basis B. If $\tilde{x}_{B}^{I} \succeq 0$, then the solution is called an intuitionistic fuzzy basic feasible solution. If $\tilde{x}_{B}^{I} \succ 0$, then the solution is called a nondegenerate intuitionistic fuzzy basic feasible solution and if atleast one component of $\tilde{x}_{B}^{I}$ is zero, then $\tilde{x}^{I}$ is called a degenerate intuitionistic fuzzy basic feasible solution.

\subsection{Properties of the IFLP Model}

Here the objective function and the constraints are all linear functions. Linearity implies that the IFLPP must satisfy the following basic properties: 


\subsubsection{Proportionality}

This property requires the contribution of each decision variable in both the objective function and the constraints to be directly proportional to the value of the variable.

\subsubsection{Additivity}

This property requires the total contribution of all the variables in the objective function and in the constraints to be the direct sum of individual contributions of each variable.

\subsubsection{Uncertainty}

In the framework of IFLPP, there are no additional assumptions about the nature of objective functions and constraints. According to different hypothesis being considered, distinct IFLPPs are obtained. In IFLP model, the coefficients in the objective function and constraints are not always deterministic. This means that any one or all of these coefficients are unknown constants - a rare occurrence in real life and moreover, the vagueness is not of probabilistic kind, the inexact values can be determined by means of models using IF numbers.

\subsubsection{Example}

Medical imaging has been an active area of research where abnormalities are detected non invasively. But dealing with medical images is a challenging task. Due to the complexity in the images, many of the structures are hardly visible; various soft computing techniques are applied by different researchers to process medical images. In order to carry out this task, intuitive ways have been found out to interpret and describe the inherent ambiguity and vagueness in the medical images in terms of intuitionistic fuzzy set theory. The efficiency of the use of IFS theory in medical image processing can be demonstrated in the context of contrast enhancement, segmentation where the performance is observed to be much better.

\section{INTUITIONISTIC FUZZY SIMPLEX METHOD}

An Intuitionistic Fuzzy Simplex Method seeks to solve a problem through a series of logical operations. Problems of the precision class are naturally restrictive and thus there arises a need for intuitionistic fuzzy algorithms applicable to IF situations.

\subsection{The Proposed IF Simplex Method in Standard Form}

The IFLPP is defined as

$$
\begin{array}{cc}
\operatorname{Max} \quad \tilde{z}^{I} \approx c \tilde{x}^{I} \\
\text { subject } \quad \text { to } \quad A \tilde{x}^{I} \preceq \tilde{b}^{I} \\
\tilde{x}^{I} \succeq 0 .
\end{array}
$$

The resource constraints in augmented form is given by

$A \tilde{x}^{I} \approx\left(\begin{array}{ll}B & N\end{array}\right)\left(\begin{array}{c}\tilde{x}_{B}^{I} \\ \tilde{x}_{N}^{I}\end{array}\right) \approx B \tilde{x}_{B}^{I}+N \tilde{x}_{N}^{I} \approx \tilde{b}^{I}$

The augmented form of the objective function is

$$
\tilde{z}^{I} \approx\left(\begin{array}{ll}
c_{B} & c_{N}
\end{array}\right)\left(\begin{array}{c}
\tilde{x}_{B}^{I} \\
\tilde{x}_{N}^{I}
\end{array}\right) \approx c_{B} \tilde{x}_{B}^{I}+c_{N} \tilde{x}_{N}^{I}
$$

Substituting (3) and (4) in (2), the given IFLPP takes the form

$$
\operatorname{Max} \tilde{z}^{I} \approx c_{B} \tilde{x}_{B}^{I}+c_{N} \tilde{x}_{N}^{I}
$$

subject to $B \tilde{x}_{B}^{I}+N \tilde{x}_{N}^{I} \approx \tilde{b}^{I}$

$$
\tilde{x}_{B}^{I}, \tilde{x}_{N}^{I} \succeq 0
$$

Then, it is possible to write

$$
\begin{gathered}
\tilde{x}_{B}^{I} \approx B^{-1} \tilde{b}^{I}-B^{-1} N \tilde{x}_{N}^{I} \quad \text { and } \\
\tilde{z}^{I} \approx c_{B}\left(B^{-1} \tilde{b}^{I}-B^{-1} N \tilde{x}_{N}^{I}\right)+c_{N} \tilde{x}_{N}^{I}
\end{gathered}
$$

The above results imply that

$$
\begin{gathered}
\tilde{x}_{B}^{I}+B^{-1} N \tilde{x}_{N}^{I} \approx B^{-1} \tilde{b}^{I} \\
\tilde{z}^{I}+\left(c_{B} B^{-1} N-c_{N}\right) \tilde{x}_{N}^{I} \approx c_{B} B^{-1} \tilde{b}^{I}
\end{gathered}
$$

Currently $\tilde{x}_{N}^{I} \approx 0$ and so $\tilde{x}_{B}^{I} \approx B^{-1} \tilde{b}^{I}$ and

the objective function $\tilde{z}^{I} \approx c_{B} B^{-1} \tilde{b}^{I}$.

The tabular format of the above IFLPP is presented in Table 1.

Table 1. IF Simplex Table

\begin{tabular}{|c|c|c|c|c|}
\hline & $\tilde{z}^{I}$ & $\tilde{x}_{B}^{I}$ & $\tilde{x}_{N}^{I}$ & R.H.S \\
\hline$\tilde{z}^{I}$ & 1 & 0 & $c_{B} B^{-1} N-c_{N}$ & $c_{B} B^{-1} \tilde{b}^{I}$ \\
\hline$\tilde{x}_{B}^{I}$ & 0 & 1 & $B^{-1} N$ & $B^{-1} \tilde{b}^{I}$ \\
\hline
\end{tabular}

Table 1 provides all the information which are needed to proceed with the IF simplex method.The IF cost row in the Table 1 is

$$
\left(\gamma_{j}\right)_{j \neq B_{j}}=\left(c_{B} B^{-1} a_{j}-c_{j}\right)_{j \neq B_{i}}=\left(z_{j}-c_{j}\right)_{j \neq B_{i}}
$$

The solution is optimal to (1) if $z_{j}-c_{j} \geq 0$ for all $1 \leq j \leq n$ and $j \neq B_{i}$. On the other hand, if $z_{l}-c_{l}<0$, for a $l \neq B_{i}$, then $\tilde{x}_{B_{r}}^{I}$ is exchanged with $\tilde{x}_{l}^{I}$. Then compute the vector $y_{l}=B^{-1} a_{l}$. If $y_{l} \leq 0$, then $\tilde{x}_{l}^{I}$ can be increased indefinitely, and then the optimal objective is unbounded. On the other hand, if $y_{l}$ has atleast one positive component, then the increase in it will be blocked by one of the current basic variables which drops to zero.

\subsection{Pivoting}

If $\tilde{x}_{l}^{I}$ enters the basis and $\tilde{x}_{B_{r}}^{I}$ leaves the basis, then pivoting on $y_{r l}$ can be stated as follows:

1) Divide row $r$ by $y_{r l}$

2) For $\mathrm{i}=1, \ldots, \mathrm{m}$ and $i \neq r$, update the $i^{\text {th }}$ row 
by adding to it $-y_{i l}$ times the new $r^{\text {th }}$ row.

3) Update row zero by adding to it $\gamma_{l}$ times the new $r^{\text {th }}$ row.

\subsection{Main Results}

\section{Theorem 5.3.1}

If the given IFLPP has a non-degenerate IF basic feasible solution

$\tilde{x}_{B}^{I} \approx B^{-1} \tilde{b}^{I}, \tilde{x}_{N}^{I} \approx 0$, then it is optimal to (1) if and only if $z_{j}-c_{j} \geq 0$ for all $1 \leq j \leq n$.

Proof

Suppose that $\tilde{x}^{* I} \approx\left(\begin{array}{c}\tilde{x}_{B}^{I} \tilde{x}_{\mathbb{N}}^{I} \\ { }^{T}\end{array}\right)^{T}$ is an IF basic feasible solution to (1) where $\tilde{x}_{B}^{I} \approx B^{-1} \tilde{b}^{I}, \tilde{x}_{N}^{I} \approx 0$. Then $\tilde{z}^{* I} \approx c_{B} \tilde{x}_{B}{ }^{I} \approx c_{B} B^{-1} \tilde{b}^{I}$. For all feasible solution $\tilde{x}^{I}$, it is possible to write, $\tilde{b}^{I} \approx A \tilde{x}^{I} \approx B \tilde{x}_{B}{ }^{I}+N \tilde{x}_{N}{ }^{I}$. Hence, the value of the objective function is given by$$
\tilde{z}^{I} \approx c \tilde{x}^{I} \approx c_{B} \tilde{x}_{B}{ }^{I}+c_{N} \tilde{x}_{N}{ }^{I}
$$$$
\approx c_{B} B^{-1} \tilde{b}^{I}-\sum_{j \neq B_{i}}\left(c_{B} B^{-1} a_{j}-c_{j}\right) \tilde{x}_{j}{ }^{I}
$$

Then $\tilde{z}^{I} \approx \tilde{z}^{* I}-\sum_{j \neq B_{i}}\left(z_{j}-c_{j}\right) \tilde{x}_{j}^{I}$

From the above result, it is clear that the solution is optimal if and only if $z_{j}-c_{j} \geq 0$ for all $1 \leq j \leq n$.

\section{Theorem 5.3.2}

If in an IF simplex table, an 1 exists such that $z_{l}-c_{l}<0$ and there exists a basic index i such that $y_{i l}>0$, then a pivoting row $\mathrm{r}$ can be found so that pivoting on $y_{r l}$ will yield an IF feasible table with a corresponding nondecreasing IF objective value.

Proof

A criterion is needed for choosing an IF basic variable to leave the basis so that the new simplex table will remain feasible and the new objective value is nondecreasing. Assume column 1 is the pivot cloumn. Also, suppose that $\tilde{x}^{I} \approx\left(\tilde{x}_{B}^{I^{T}} \tilde{x}_{N}^{I}{ }^{T}\right)^{T}$ is an IF basic feasible solution to the IFLPP, where $\tilde{x}_{B}^{I} \approx B^{-1} \tilde{b}^{I}$ and $\tilde{x}_{N} \approx 0$. Then the corresponding IF objective value is $\tilde{z}^{I} \approx c_{B} B^{-1} \tilde{b}^{I} \approx c_{B} \tilde{y}_{0}{ }^{I}$. For any IF basic feasible solution to the IFLPP, it is possible to write $\tilde{x}_{B}{ }^{I}+\sum_{j \neq B_{i}} y_{j} \tilde{x}_{j}{ }^{I} \approx \tilde{y}_{0}{ }^{I}$ where $y_{j}=B^{-1} a_{j}$.

So, if $\tilde{x}_{l}{ }^{I}$ enters into the basis, then $\tilde{x}_{B}{ }^{I} \approx \tilde{y}_{0}{ }^{I}-y_{l} \tilde{x}_{l}{ }^{I}$. As $\tilde{x}_{B}{ }^{I}$ is assumed to be feasible, it is clear that $\tilde{y}_{i 0}{ }^{I}-y_{i l} \tilde{x}_{l}{ }^{I} \succeq 0$, for all $\mathrm{i}=1, \ldots, \mathrm{m}$. If $y_{i l} \leq 0$, then it is obvious that the above condition holds good. Hence for all $y_{i l}>0$, it is necessary to have $\tilde{x}_{l}^{I} \preceq \frac{\tilde{y}_{i 0}{ }^{I}}{y_{i l}}$.

To satisfy the above condition, it is sufficient to let $\frac{\tilde{y}_{r 0}^{I}}{y_{r l}} \approx \min \left\{\frac{\tilde{y}_{i 0}^{I}}{y_{i l}} / y_{i l}>0\right\}$.

Also, for any IF basic feasible solution to the IFLPP, the value of the objective function is given by

$\tilde{z}^{I} \approx c_{B} \tilde{y}_{0}{ }^{I}-\sum_{j \neq B_{i}}\left(z_{j}-c_{j}\right) \tilde{x}_{j}{ }^{I}$.

If $\tilde{x}_{l}^{I}$ is entered into the basis, then $\tilde{z}^{I} \approx c_{B} \tilde{y}_{0}{ }^{I}-\left(z_{l}-c_{l}\right) \tilde{x}_{l}{ }^{I}$. It is clear that the new objective value is nondecreasing since $\tilde{z}^{I} \approx c_{B} \tilde{y}_{0}{ }^{I}-\left(z_{l}-c_{l}\right) \tilde{x}_{l}{ }^{I} \succeq c_{B} \tilde{y}_{0}{ }^{I}$ using the fact that $\left(z_{l}-c_{l}\right) \tilde{x}_{l}^{I} \preceq 0$.

\section{Theorem 5.3.3}

If for any intuitionistic fuzzy basic feasible solution to the IFLPP, in $l^{\text {th }}$ column, not in basis, for which $z_{l}-c_{l}<0$ and $y_{i l} \leq 0$, for every $\mathrm{i}=1, \ldots, \mathrm{m}$, then the IFLPP has an unbounded solution.

Proof

If $\tilde{x}_{B}{ }^{I}$ is assumed to be an IF basic solution to the IFLPP, then

$\tilde{x}_{B_{i}}{ }^{I}+\sum_{j \neq B_{i}} y_{i j} \tilde{x}_{j}{ }^{I} \approx \tilde{y}_{i 0}{ }^{I}, \mathrm{i}=1, \ldots, \mathrm{m} ; \mathrm{j}=1, \ldots, \mathrm{n}$
$\Rightarrow \tilde{x}_{B_{i}}{ }^{I} \approx \tilde{y}_{i 0}{ }^{I}-\sum_{j \neq B_{i}} y_{i j} \tilde{x}_{j}{ }^{I}, \mathrm{i}=1, \ldots, \mathrm{m}, \mathrm{j}=1, \ldots, \mathrm{n}$.

If the variable $\tilde{x}_{l}{ }^{I}$ is entered into the basis, then $\tilde{x}_{l}{ }^{I} \succ 0$ and $\tilde{x}_{j}{ }^{I} \approx 0$, for all $j \neq B_{i} \cup l$. Since $y_{i l} \leq 0$, $\mathrm{i}=1, \ldots, \mathrm{m}$, it is clear that $\tilde{y}_{i 0}{ }^{I}-y_{i l} \tilde{x}_{l}{ }^{I} \succeq 0$. This implies that $\tilde{x}_{B}{ }^{I} \geq 0$. Therefore, the current intuitionistic fuzzy basic solution will remain feasible.

Now, the value of $\hat{z}$ for the above IF feasible solution is as follows: $\quad \hat{z} \approx c_{B} \tilde{x}_{B}{ }^{I}+c_{N} \tilde{x}_{N}{ }^{I}$

$$
\begin{aligned}
& \approx \sum_{i=1}^{m} c_{B_{i}}\left(\tilde{y}_{i 0}{ }^{I}-y_{i l} \tilde{x}_{l}{ }^{I}\right)+c_{l} \tilde{x}_{l}{ }^{I} \\
& \approx \sum_{i=1}^{m} c_{B_{i}} \tilde{y}_{i 0}{ }^{I}-\left(\sum_{i=1}^{m} c_{B_{i}} y_{i l}-c_{l}\right) \tilde{x}_{l}{ }^{I} \\
& \approx c_{B} \tilde{y}_{0}{ }^{I}-\left(c_{B} y_{l}-c_{l}\right) \tilde{x}_{l}{ }^{I} \\
& \approx \tilde{z}^{I}-\left(z_{l}-c_{l}\right) \tilde{x}_{l}{ }^{I}
\end{aligned}
$$

So, $\hat{z}=\tilde{z}^{I}-\left(z_{l}-c_{l}\right) \tilde{x}_{l}^{I}$.

From the above result, it is clear that the solution is unbounded as the variable $\tilde{x}_{l}{ }^{I}$ can be entered into the basis with arbitrarily large intuitionistic fuzzy value. 


\subsection{The Proposed Intuitionistic Fuzzy Simplex Algorithm}

For the solution of any IFLPP by IF simplex algorithm, the existence of an initial IF basic feasible solution is always assumed.

The steps for the computation of an optimum solution are as follows:

Step1

Check whether the objective function of the given IFLPP is to be maximized or minimized. It it is to be minimized, then it is converted into a problem of maximizing it by using the result

Minimum $z=-\operatorname{Maximum}(-z)$

Step 2

Convert all the inequations of the constraints into equations by introducing slack and/or surplus variables in the constraints. Put the costs of these variables equal to zero.

Step 3

Obtain an initial intuitionistic fuzzy basic feasible solution to the problem in the form $\tilde{x}_{B}{ }^{I} \approx B^{-1} \tilde{b}^{I} \approx \tilde{y}_{0}{ }^{I}$ and $\tilde{x}_{N}{ }^{I} \approx 0$.

The intuitionistic fuzzy objective is $\tilde{z}^{I} \approx c_{B} B^{-1} \tilde{b}^{I} \approx c_{B} \tilde{y}_{0}^{I}$.

Step 4

Calculate $w=c_{B} B^{-1}$ and $y_{0}=\mathfrak{R}\left(\tilde{y}_{0}{ }^{I}\right)$. For each non basic variable calculate $\gamma_{j}=z_{j}-c_{j}=c_{B} B^{-1} a_{j}-c_{j}$ $=w a_{j}-c_{j}$. Let $\gamma_{l}=\min \left\{\gamma_{j}\right\}$ where $1 \leq j \leq n$. If $\gamma_{l} \geq 0$, then stop the procedure and the current solution is optimal. Otherwise go to step 5 .

\section{Step 5}

Calculate $y_{l}=B^{-1} a_{l}$. If $y_{l} \leq 0$, then stop; the optimal solution is unbounded. Otherwise determine the index of the variable $\tilde{x}_{B_{r}}{ }^{I}$ leaving the basis as follows: $\frac{y_{r 0}}{y_{r l}}=\min \left\{\frac{y_{i 0}}{y_{i l}} / y_{i l}>0 \quad\right.$ and $\left.\quad 1 \leq i \leq m\right\} \quad$ Update $\tilde{y}_{i 0}{ }^{I}$ by replacing $\tilde{y}_{i 0}{ }^{I}-\frac{\tilde{y}_{r 0}{ }^{I}}{y_{r l}} y_{i l}$ for $i \neq r$ and $\tilde{y}_{r 0}{ }^{I}$ by replacing $\frac{\tilde{y}_{r 0}^{I}}{y_{r l}}$. Also, update $\tilde{z}^{I}$ by replacing $\tilde{z}^{I}-\frac{\tilde{y}_{r 0}^{I}}{y_{r l}}\left(z_{l}-c_{l}\right)$.

Step 6

Update B by replacing $a_{B_{r}}$ with $a_{l}$ and go to step 4 and repeat the procedure until the optimality is reached.

\subsection{A Numerical Example}

An example is presented to depict the working of the proposed IF simplex algorithm technique for solving

\section{IFLPP.}

Reddy Mikks produces both interior and exterior paints from two raw materials, $\mathbf{M}_{1}$ and $\mathbf{M}_{2}$. The following table provides the required data of the problem:

Table 2

\begin{tabular}{|c|c|c|}
\hline & \multicolumn{2}{|c|}{$\begin{array}{c}\text { Tons of raw material per ton } \\
\text { of }\end{array}$} \\
\hline & $\begin{array}{c}\text { Exterior } \\
\text { paint }\end{array}$ & $\begin{array}{c}\text { Interior } \\
\text { paint }\end{array}$ \\
\hline $\begin{array}{c}\text { Raw material } \\
\mathrm{M}_{1}\end{array}$ & 6 & 4 \\
\hline $\begin{array}{c}\text { Raw material } \\
\mathrm{M}_{2}\end{array}$ & 1 & 2 \\
\hline Profit per ton & 5 & 4 \\
\hline
\end{tabular}

It is noted that maximum daily availability of raw materials can vary from day to day due to breakdown of machines, delay supply, etc. At the same time, the maximum daily availability is some what close to 24 tons for $\mathrm{M}_{1}$ and 6 tons for $\mathrm{M}_{2}$. Also, a market survey indicates that the daily demands of exterior and interior paints can also vary due to variations in the requirement of consumers. But it is expected that the daily demand for interior paint can not exceed that for exterior paint by more than 4 tons approximately. Also, the maximum daily demand for interior paint is some what close to 2 tons.

Reddy Mikks wants to determine the optimum (best) product mix of interior and exterior paints that maximizes the total daily profit.For the Reddy Mikks problem, it is necessary to determine the daily amounts to be produced of exterior and interior paints.

Since the maximum daily availability of raw materials $\mathrm{M}_{1}$ and $\mathrm{M}_{2}$ are uncertain, the daily amounts to be produced of exterior and interior paints will also be uncertain. So, the problem can be modelled as an intuitionistic fuzzy linear programming problem by using symmetric trapezoidal intuitionistic fuzzy numbers for each uncertain value and solved using arithmetic operations on STIFN [15].

Maximum daily availability for raw material $\mathrm{M}_{1}$ which is close to 24 can be modelled as $[23,25,1,1 ; 23,25,3,3]$.

Similarly, the other parameters are also modelled as STIFNs taking into account the nature of the problem and other requirements.

The given IFLPP can be formulated as

$$
\operatorname{Max} \tilde{z}^{I}=5 \tilde{x}_{1}^{I}+4 \tilde{x}_{2}{ }^{I}
$$

subject to $6 \tilde{x}_{1}{ }^{I}+4 \tilde{x}_{2}{ }^{I} \preceq[23,25,1,1 ; 23,25,3,3]$

$\tilde{x}_{1}{ }^{I}+2 \tilde{x}_{2}{ }^{I} \preceq[5,7,2,2 ; 5,7,4,4]$

$-\tilde{x}_{1}{ }^{I}+\tilde{x}_{2}{ }^{I} \preceq[3,5,4,4 ; 3,5,6,6]$

$$
\tilde{x}_{2}{ }^{I} \preceq[1,3,2,2 ; 1,3,4,4]
$$

and $\tilde{x}_{1}{ }^{I}, \tilde{x}_{2}{ }^{I} \succeq 0$.

Now, the standard form of the intuitionistic fuzzy linear programming problem becomes 


$$
\operatorname{Max} \tilde{z}^{I}=5 \tilde{x}_{1}^{I}+4 \tilde{x}_{2}^{I}
$$

subject to $6 \tilde{x}_{1}^{I}+4 \tilde{x}_{2}{ }^{I}+\tilde{x}_{3}^{I} \approx$

$[23,25,1,1 ; 23,25,3,3]$

$\tilde{x}_{1}{ }^{I}+2 \tilde{x}_{2}{ }^{I}+\tilde{x}_{4}{ }^{I} \approx[5,7,2,2 ; 5,7,4,4]$

$-\tilde{x}_{1}{ }^{I}+\tilde{x}_{2}{ }^{I}+\tilde{x}_{5}{ }^{I} \approx[3,5,4,4 ; 3,5,6,6]$

$$
\tilde{x}_{2}{ }^{I}+\tilde{x}_{6}{ }^{I} \approx[1,3,2,2 ; 1,3,4,4]
$$

$\tilde{x}_{1}^{I}, \tilde{x}_{2}{ }^{I}, \tilde{x}_{3}{ }^{I}, \tilde{x}_{4}{ }^{I}, \tilde{x}_{5}{ }^{I}, \tilde{x}_{6}{ }^{I} \succeq 0$.

where $\tilde{x}_{3}{ }^{I}, \tilde{x}_{4}{ }^{I}, \tilde{x}_{5}{ }^{I}$ and $\tilde{x}_{6}{ }^{I}$ are the slack intuitionistic fuzzy variables.

The initial basic feasible solution is presented in Table 3 .

Table 3

\begin{tabular}{|c|c|c|c|c|c|c|c|c|}
\hline Basis $\left(\tilde{x}_{B}{ }^{I}\right)$ & $\tilde{x}_{1}{ }^{I}$ & $\tilde{x}_{2}{ }^{I}$ & $\tilde{x}_{3}{ }^{I}$ & $\tilde{x}_{4}{ }^{I}$ & $\tilde{x}_{5}{ }^{I}$ & $\tilde{x}_{6}{ }^{I}$ & R.H.S & $\Re$ \\
\hline$\tilde{x}_{3}{ }^{I}$ & $6^{*}$ & 4 & 1 & 0 & 0 & 0 & {$[23,25,1,1 ; 23,25,3,3]$} & 49 \\
\hline$\tilde{x}_{4}{ }^{I}$ & 1 & 2 & 0 & 1 & 0 & 0 & {$[5,7,2,2 ; 5,7,4,4]$} & 13 \\
\hline$\tilde{x}_{5}{ }^{I}$ & -1 & 1 & 0 & 0 & 1 & 0 & {$[3,5,4,4 ; 3,5,6,6]$} & 9 \\
\hline$\tilde{x}_{6}{ }^{I}$ & 0 & 1 & 0 & 0 & 0 & 1 & {$[1,3,2,2 ; 1,3,4,4]$} & 5 \\
\hline$z_{j}-c_{j} \Rightarrow$ & 0 & 0 & -5 & -4 & 0 & 0 & $\tilde{0}^{I}$ & 0 \\
\hline
\end{tabular}

From Table 3, it is obtained that $\gamma_{3}=z_{3}-c_{3}=-5<0$ and $\gamma_{4}=z_{4}-c_{4}=-4<0$. Hence the intuitionistic fuzzy nonbasic variable $\tilde{x}_{1}^{I}$ is an entering variable. Therefore, according to the minimum ratio test given in the step 5 of the IF simplex algorithm, $\tilde{x}_{3}{ }^{I}$ is a leaving variable. Now, after pivoting, the new table is given in Table 4.

Table 4

\begin{tabular}{|c|c|c|c|c|c|c|c|c|}
\hline $\operatorname{Basis}\left(\tilde{x}_{B}{ }^{I}\right)$ & $\tilde{x}_{1}{ }^{I}$ & $\tilde{x}_{2}{ }^{I}$ & $\tilde{x}_{3}{ }^{I}$ & $\tilde{x}_{4}{ }^{I}$ & $\tilde{x}_{5}{ }^{I}$ & $\tilde{x}_{6}{ }^{I}$ & R.H.S & $\Re$ \\
\hline$\tilde{x}_{1}{ }^{I}$ & 1 & $\frac{2}{3}$ & $\frac{1}{6}$ & 0 & 0 & 0 & {$\left[\frac{23}{6}, \frac{25}{6}, \frac{1}{6}, \frac{1}{6} ; \frac{23}{6}, \frac{25}{6}, \frac{3}{6}, \frac{3}{6}\right]$} & $\frac{49}{6}$ \\
\hline$\tilde{x}_{4}{ }^{I}$ & 0 & $\frac{4^{*}}{3}$ & $\frac{-1}{6}$ & 1 & 0 & 0 & {$\left[\frac{5}{6}, \frac{19}{6}, \frac{13}{6}, \frac{13}{6} ; \frac{5}{6}, \frac{19}{6}, \frac{27}{6}, \frac{27}{6}\right]$} & $\frac{31}{6}$ \\
\hline$\tilde{x}_{5}{ }$ & 0 & $\frac{5}{3}$ & $\frac{1}{6}$ & 0 & 1 & 0 & {$\left[\frac{41}{6}, \frac{55}{6}, \frac{25}{6}, \frac{25}{6} ; \frac{41}{6}, \frac{55}{6}, \frac{39}{6}, \frac{39}{6}\right]$} & $\frac{103}{6}$ \\
\hline$\tilde{x}_{6}{ }^{I}$ & 0 & 1 & 0 & 0 & 0 & 1 & {$[1,3,2,2 ; 1,3,4,4]$} & 5 \\
\hline$z_{j}-c_{j} \Rightarrow$ & 0 & $\frac{-2}{3}$ & $\frac{5}{6}$ & 0 & 0 & 0 & {$\left[\frac{115}{6}, \frac{125}{6}, \frac{5}{6}, \frac{5}{6} ; \frac{115}{6}, \frac{125}{6}, \frac{15}{6}, \frac{15}{6}\right]$} & $\frac{245}{6}$ \\
\hline
\end{tabular}

Hence the intuitionistic fuzzy nonbasic variable $\tilde{x}_{2}{ }^{I}$ is an entering variable. Therefore, according to the minimum ratio test given in the step 5 of the IF simplex algorithm, $\tilde{x}_{4}{ }^{I}$ is a leaving variable. Now, after pivoting, the new table is given in Table 5 . 
Table 5

\begin{tabular}{|c|c|c|c|c|c|c|c|c|}
\hline $\operatorname{Basis}\left(\tilde{x}_{B}{ }^{I}\right)$ & $\tilde{x}_{1}^{I}$ & $\tilde{x}_{2}{ }^{I}$ & $\tilde{x}_{3}{ }^{I}$ & $\tilde{x}_{4}{ }^{I}$ & $\tilde{x}_{5}{ }^{I}$ & $\tilde{x}_{6}{ }^{I}$ & R.H.S & $\mathfrak{R}$ \\
\hline$\tilde{x}_{1}^{I}$ & 1 & 0 & $\frac{1}{4}$ & $\frac{-1}{2}$ & 0 & 0 & {$\left[\frac{9}{4}, \frac{15}{4}, \frac{5}{4}, \frac{5}{4} ; \frac{9}{4}, \frac{15}{4}, \frac{11}{4}, \frac{11}{4}\right]$} & $\frac{27}{4}$ \\
\hline$\tilde{x}_{2}{ }^{I}$ & 0 & 1 & $\frac{-1}{8}$ & $\frac{3}{4}$ & 0 & 0 & {$\left[\frac{5}{8}, \frac{19}{8}, \frac{13}{8}, \frac{13}{8} ; \frac{5}{8}, \frac{19}{8}, \frac{27}{8}, \frac{27}{8}\right]$} & $\frac{31}{8}$ \\
\hline$\tilde{x}_{5}{ }^{I}$ & 0 & 0 & $\frac{9}{24}$ & $\frac{-5}{4}$ & 1 & 0 & {$\left[\frac{23}{8}, \frac{65}{8}, \frac{55}{8}, \frac{55}{8} ; \frac{23}{8}, \frac{65}{8}, \frac{97}{8}, \frac{97}{8}\right]$} & $\frac{109}{8}$ \\
\hline$\tilde{x}_{6}{ }^{I}$ & 0 & 0 & $\frac{1}{8}$ & $\frac{-3}{4}$ & 0 & 1 & {$\left[\frac{-11}{8}, \frac{19}{8}, \frac{29}{8}, \frac{29}{8} ; \frac{-11}{8}, \frac{19}{8}, \frac{59}{8}, \frac{59}{8}\right]$} & $\frac{23}{8}$ \\
\hline$z_{j}-c_{j} \Rightarrow$ & 0 & 0 & $\frac{3}{4}$ & $\frac{1}{2}$ & 0 & 0 & {$\left[\frac{55}{4}, \frac{113}{4}, \frac{51}{4}, \frac{51}{4} ; \frac{55}{4}, \frac{113}{4}, \frac{109}{4}, \frac{109}{4}\right.$} & $\frac{197}{4}$ \\
\hline
\end{tabular}

From Table 5, for the intuitionistic nonbasic variables $\tilde{x}_{3}{ }^{I}$ and $\tilde{x}_{4}{ }^{I}$, it is found that $\gamma_{3}=\frac{3}{4}>0$ and $\gamma_{4}=\frac{1}{2}>0$.

Therefore, the optimal solution is given by $\tilde{x}_{1}^{I} \approx\left[\frac{9}{4}, \frac{15}{4}, \frac{5}{4}, \frac{5}{4} ; \frac{9}{4}, \frac{15}{4}, \frac{11}{4}, \frac{11}{4}\right]$ and $\mathfrak{R}\left[\tilde{x}_{1}^{I}\right]=$ 6.75 tons and $\tilde{x}_{2}{ }^{I} \approx\left[\frac{5}{8}, \frac{19}{8}, \frac{13}{8}, \frac{13}{8} ; \frac{5}{8}, \frac{19}{8}, \frac{27}{8}, \frac{27}{8}\right]$ and $\mathfrak{R}\left[\tilde{x}_{2}{ }^{I}\right]=3.875$ tons with maximum of $\tilde{z}^{I} \approx\left[\frac{55}{4}, \frac{113}{4}, \frac{51}{4}, \frac{51}{4} ; \frac{55}{4}, \frac{113}{4}, \frac{109}{4}, \frac{109}{4}\right] \quad$ with $\Re\left[\tilde{z}^{I}\right]=\frac{197}{4}=49.25$ tons.

Reddy Mikks must produce 6.75 tons of exterior paint and 3.875 tons of interior paint in order to maximize the total daily profit and the daily maximum profit is 49.25 tons.

\subsection{Interpretation of IF Simplex Method}

Though the existing methods have been extensively used to capture uncertainty and vaguness in fuzzy linear programming problems, there is no way to model hesitancy. Intuitionistic fuzzy simplex method, a new method of solving IFLPP addresses this issue of uncertainty. This approach gives a complete choice of alternatives to the decision maker in an IF environment.

Moreover, the solution procedure explained in the IF simplex method is easier than that of existing methods.

\section{CONCLUSION}

In this study, a general model of IFLPP with STIFN has been introduced and an IF simplex algorithm has been proposed to solve the problem without converting it to its crisp equivalent. The algorithm presents a credible selective and allocative tool for the optimal consumption of the constrained resources. STIFNs are ranked with a special ranking function keeping central thought that the same has to be used to solve a class of linear programming problems in which the data parameters are STIFNs. The solution methodology for such a class of IFLPPs is illustrated through an example. Also, this approach can easily be extended to more experiment and industrial applications concerning decision making in an uncertain environment.

\section{ACKNOWLEDGMENT}

The author R.Parvathi would like to thank the University Grants Commission, New Delhi, India for its financial support to UGC Research Award No. F. 30 - 1/ 2009 ( SA - II ) dated $2^{\text {nd }}$ July 2009.

\section{REFERENCES}

[1] R.E. Bellman and L.A. Zadeh, "Decision making in a fuzzy environment", Management Science 17(1970), 141-164.

[2] H. Tanaka and K.Asai, "Fuzzy linear programming problems with fuzzy numbers", Fuzzy Sets and Systems, 13(1984), 1-10.

[3] H.J. Zimmermann, "Fuzzy programming and linear programming with several objective functions", Fuzzy Sets and Systems, 1(1978), 45-55.

[4] M. Jimenez, "Ranking fuzzy numbers through the comparision of its expected intervals", International Journal of Uncertainty, Fuzziness and KnowledgeBased Systems, 4(1996), 379-388.

[5] H.R. Maleki, "Ranking functions and their applications to fuzzy linear programming", Far East Journal of Mathematical Sciences, 4(2002), 283-301.

[6] X. Wang and E.E. Kerre, "Reasonable properties for the ordering of fuzzy quantities(i)", Fuzzy Sets and Sytems, 118(2001), 375-385.

[7] K.T. Atanassov, 1999, Intuitionistic Fuzzy Sets: Theory and Applications, Physica-Verlag, Heidelberg, New York.

[8] H. Bustine and P. Burillo, "Vague sets are intuitionistic fuzzy sets", Fuzzy Sets and Systems 79(1996), 403-405.

[9] H.B. Mitchell, "Ranking-intuitionistic fuzzy 
numbers", International Journal of Uncertainty, Fuzziness and Knowledge Based Systems, 12, 3(2004), 377-386.

[10] A. Kumar, J. Kaur and P. Singh, "A new method for solving fully fuzzy linear programming problems", Applied Mathematical Modeling, 35(2011), 817-823.

[11] H.R. Maleki, M. Tata and M. Mashinchi, "Linear programming with fuzzy variables", Fuzzy Sets and Systems , 109(2000), 21-33.

[12]K. Ganesan and P. Veeramani, "Fuzzy linear programs with trapezoidal fuzzy numbers", Ann.Oper.Res.143(2006), 305-315.
[13]B.S. Mahapatra and G.S. Mahapatra, "Intuitionistic fuzzy fault tree analysis using intuitionistic fuzzy numbers", International Mathematical Forum, 5, 21(2010), $1015-1024$

[14] H.M. Nehi, "A new ranking method for intuitionstic fuzzy numbers", International Journal of Fuzzy Systems, 12, 1(2010), 80-86.

[15] R. Parvathi and C. Malathi, "Arithmetic operations on symmetric trapezoidal intuitionistic fuzzy numbers", International Journal of Soft Computing and Engineering, 2, 2(2012), 268-273. 